\title{
Morphologic Changes in Esophageal Body Movement During Bolus Transport After Peroral Endoscopic Myotomy in Type III Achalasia
}

\author{
Alex Ju Sung Kim, ${ }^{1}$ Sungmoon Ong, ${ }^{2}$ Ji Hyun Kim, ${ }^{2,3 *}$ Hong Sub Lee, ${ }^{2}$ Jun Sik Yoon, ${ }^{2}$ and Dae Young Hur ${ }^{1}$ \\ ${ }^{1}$ Department of Anatomy and Tumor Immunology, Inje University College of Medicine, Busan, Korea; ${ }^{2}$ Department of Internal Medicine, Busan \\ Paik Hospital, Inje University College of Medicine, Busan, Korea; and ${ }^{3}$ Department of Internal Medicine, Good Gangan Hospital, Busan, Korea
}

\section{Background/Aims}

The effect of peroral endoscopic myotomy (POEM) on esophageal body movement in achalasia is poorly understood. This study aims to evaluate morphological changes in esophageal body movement after POEM in type III achalasia by analyzing intraluminal ultrasound (US) images in comparison to type I and II achalasia.

\section{Methods}

Intraluminal US images and impedance values of the distal esophagus from 47 achalasia patients who underwent POEM or pneumatic dilatation (PD) (30 patients in the POEM group and 17 patients in the PD group) with pre- and post-procedural high-resolution impedance manometry and intraluminal US examinations were analyzed. The muscle thickness (MT), muscle cross-sectional area, lumen cross-sectional area (LCSA), contractility and distensibility indices, swallow-to-distension interval, and distension duration during each bolus transport were analyzed.

\section{Results}

The MT increased and LCSA decreased significantly $(P<0.001)$, but the contractility index was not improved after POEM or PD in type I achalasia. Baseline MT increased and LCSA decreased significantly after POEM and PD in type II achalasia $(P<0.001)$. In contrast, MT and the swallow-to-distension interval decreased and the distension LCSA/duration and contractility index increased after POEM in type III achalasia $(P<0.001)$. In contrast to type I and II achalasia, in type III achalasia, these effects were unique to the POEM group.

\section{Conclusions}

POEM decreased the esophageal LCSA by decreasing intrabolus pressure without improving contractility in type I and II achalasia. In contrast, POEM increased esophageal body distension and contractility and improved the inhibitory process during bolus transport in type III achalasia.

(J Neurogastroenterol Motil 2022;28:131-144)

\section{Key Words}

Body movement; Esophageal achalasia; Myotomy; Ultrasonography

Received: January 24, 2021 Revised: February 28, 2021 Accepted: March 21, 2021

(.) This is an Open Access article distributed under the terms of the Creative Commons Attribution Non-Commercial License (http://creativecommons. org/licenses/by-nc/4.0) which permits unrestricted non-commercial use, distribution, and reproduction in any medium, provided the original work is properly cited.

*Correspondence: Ji Hyun Kim, MD, PhD

Department of Internal Medicine, Good Gangan Hospital, 493 Suyeong-ro, Suyeong-Gu, Busan 48265, Korea Tel: +82-51-610-9017, Fax: +82-51-955-2774, E-mail: zep202020@gmail.com 


\section{Introduction}

Due to the development of diagnostic modalities for esophageal motility disorders, achalasia can now be subclassified into 3 subtypes according to the pattern of esophageal body pressurization. ${ }^{1}$ Recent histologic reports also support the conceptual model explaining different morphologic phenotypes of achalasia, ie, that the pathophysiology of type I and II achalasia is basically loss of inhibitory neurons but that of type III achalasia is distinguished by imbalance between postganglionic inhibitory and excitatory neurons. ${ }^{2,3}$ In fact, type III achalasia was reported to be associated with poor treatment response to pneumatic dilatation (PD) and Heller myotomy, ${ }^{4-6}$ and this may be due to inhibitory function disorders involving the distal esophageal body as well as the lower esophageal sphincter (LES).

Peroral endoscopic myotomy (POEM), which has advantages over PD, exact myotomy of the LES in a tailored manner under direct visualization, and long distal esophageal myotomy was introduced as an effective alternative treatment option for achalasia. Most recent comparative clinical studies have also reported superior long-term outcomes of POEM over PD. ${ }^{7,8}$ Additionally, POEM has been reported to be especially effective for type III achalasia and cases of failure or recurrence after PD or Heller myotomy. ${ }^{9,10}$

Based on previous studies, we can hypothesize that in addition to decreasing LES pressure, POEM can also modify esophageal body movement in achalasia, and the effect may be distinguishable in type III achalasia compared to type I and II achalasia. The answer to this hypothesis will provide useful information for deciding the treatment strategy for achalasia, such as the length of myotomy, as well as for providing essential evidence for applying POEM in spastic esophageal motility disorders other than type III achalasia, such as jackhammer esophagus and distal esophageal spasm, in which LES relaxation during primary peristalsis is intact and the main pathophysiology is confined to the esophageal body. ${ }^{11} \mathrm{Howev-}$ er, studies on changes in esophageal body movement after POEM are limited $^{12}$ and based on timed barium esophagography, which provides indirect information about esophageal bolus transit. ${ }^{13}$ The recently introduced functional imaging probe was reported to provide clinically useful information about esophageal distensibility after POEM, but it has limitations in providing primary peristalsis data. $^{14}$

Although it is labor intensive, intraluminal ultrasound (US) examination has strong advantages in terms of providing detailed information on cross-sectional esophageal body movement and inhibitory function during primary peristalsis by directly measuring changes in esophageal body muscle thickness and muscle/lumen cross-sectional area (CSA). ${ }^{15,16}$

Therefore, we performed this study to evaluate the morphologic changes in cross-sectional movement in the esophageal body after POEM in type III achalasia in comparison to type I and II achalasia and to those after PD by analyzing intraluminal US images temporally correlated with high-resolution impedance manometry (HRIM) data.

\section{Materials and Methods}

\section{Patients and Healthy Controls}

The medical records of consecutive achalasia patients aged 18 to 80 who underwent POEM or PD at a tertiary referral center from March 2014 to December 2019 were retrospectively reviewed.

The inclusion criteria were as follows: (1) confirmed primary achalasia based on HRIM according to the Chicago classification, version $3.0{ }^{1}{ }^{2}$ (2) intraluminal US performed with a $20-\mathrm{MHz}$ miniprobe catheter simultaneously with HRIM; (3) POEM or PD performed as the initial therapeutic modality within 3 months of the initial diagnosis; and (4) follow-up HRIM and intraluminal US performed simultaneously within 3-6 months after the procedure.

The exclusion criteria were as follows: (1) clinical outcome data not available at least 1 year after POEM or PD; (2) treatment failure, which was defined as no improvement or relapse of symptoms, with an increase in the Eckart score to 4 or higher within a month.; and (3) US images that were too poor for parameter analysis.

To validate the intraluminal US and impedance parameters, 8 healthy controls were also included in the study.

\section{Simultaneous High-resolution Impedance Manometry and Intraluminal Ultrasound Image Recording}

All studies were conducted using a solid-state HRIM catheter equipped with 36 pressure sensors and 18 impedance channels (MSC-3890-Z, Given Imaging, Los Angeles, CA, USA). Subjects were positioned in the $20^{\circ}$ low Fowler's position, and data were acquired during swallows of $10 \mathrm{~mL}$ of $0.5 \mathrm{~N}$ saline spaced at 30 -second intervals. Swallows were repeated at least 10 times. Dedicated software (ManoView, Given Imaging, CA, USA) was used to visualize the plots and calculate the metrics. Achalasia was classified into 3 subtypes according to the Chicago classification, version 3.0 . 
Intraluminal US examination was performed simultaneously with HRIM examination using a $20-\mathrm{MHz}$ miniprobe catheter (UM3D-DP20-25R, Olympus, Tokyo, Japan), which was interfaced with an EU-ME2 system (Olympus), and simultaneous esophageal pressures, US images and intraluminal impedance signals were acquired. The HRIM and US catheters were introduced separately through each nostril, and the US transducer was positioned to acquire images at the distal esophagus, $5 \mathrm{~cm}$ proximal to the LES.

Simultaneous HRIM and intraluminal US examinations were performed initially and repeated between 3 and 6 months after POEM or PD.

\section{Data Extraction and Analysis}

US images of 3 to 5 analyzable swallows from each examination were captured from the DVD files with Adobe Premier CS 3.0 (Adobe Systems, Mountain View, CA, USA) and then converted to BMP tomographic B-mode images at 30 frames per second. The B-mode US images were converted into 16 equispaced Mmode images (every $22.5^{\circ}$ apart). ${ }^{17}$

To evaluate the cross-sectional contractility and distensibility of the esophageal body during bolus transit, muscle thickness (MT), muscle CSA (MCSA), and lumen CSA (LCSA) were calculated using B-mode US images. The contractility index (defined as [distension LCSA - contraction LCSA]/distension LCSA) and distensibility index (defined as distension LCSA/baseline LCSA) of each bolus transport cycle were also analyzed.

To evaluate deglutitive inhibitory function as well as distensibility, the swallow-to-distension interval (which was defined as the time between swallowing and the onset of esophageal distension) and distension duration (which was defined as the time between the onset of esophageal distension and closure of the esophageal lumen) were analyzed using M-mode US images in type III achalasia.

The B-mode US images of each swallow used to analyze the baseline/contraction/distension MT, MCSA, and LCSA were selected using M-mode US images. Image analysis was performed using Sigma Scan Pro 5 (Jandel Scientific, San Rafael, CA, USA).

Baseline MT/MCSA/LCSA values were calculated from Bmode images captured 0.5-1 seconds before swallowing. Distension MT/MCSA/LCSA values were obtained from the B-mode images captured at peak luminal distension during bolus transit. Contraction MT/MCSA/LCSA values were obtained from B-mode images showing the point at which the muscle layer became thickest with accompanying lumen occlusion/decrease during bolus transit.

The average distance of the 4 points, including the thinnest and the thickest points, parallel to the US beam between the outer longitudinal muscle edge and inner margin of the inner circular muscle represents the MT. The MCSA was measured by subtracting the CSA from the inner rings of the esophageal muscle from the total esophageal CSA.

The LCSA was obtained by marking the interface between the liquid and the inner margin of the esophageal mucosa. The impedance value of the distal esophagus $5 \mathrm{~cm}$ proximal to the LES and M-mode US images were temporally correlated to obtain baseline and nadir impedance values. To doublecheck the change in the LCSA before and after the procedure, the baseline luminal impedance value, defined as the average value 1 second before the onset of swallowing, was calculated in all subjects. The nadir luminal impedance value, which corresponded to the peak distension point, was also obtained using temporally correlated impedance values and Mmode US images in type III achalasia patients and healthy controls (Fig. 1).

Along with cross-sectional image analysis, pre- and post-procedural changes in esophageal peristalsis parameters, such as completeness and sequentiality of bolus transport and panesophageal pressurization (PEP), and HRIM parameters, such as 4-second integrated relaxation pressure (4s-IRP) and basal LES pressure, were also analyzed.

The pattern of bolus transport and completion of bolus clearance was defined based on the analysis of the impedance waveform. For bolus transport to be defined as sequential, the impedance waveform was required to meet the following 2 criteria: (1) the onset of the decrease in the impedance waves progressed in the aboral direction and (2) the velocity of onset of the decrease in impedance wave from the mid-esophagus to the distal esophagus just above LES was less than $8 \mathrm{~cm} / \mathrm{second}$. Complete bolus clearance was defined as the return of esophageal impedance to $50 \%$ of its baseline value. ${ }^{18} \mathrm{PEP}$ was defined as uniform pressurization of $>$ $30 \mathrm{mmHg}$ extending from the upper esophageal sphincter to the esophagogastric junction.

\section{Peroral Endoscopic Myotomy and Pneumatic Dilatation Technique}

POEM was performed under general anesthesia. The orientation of the mucosal incision and subsequent submucosal tunneling and myotomy were performed in a posterior fashion. A submucosal tunnel was established 2-3 cm beyond the LES. Subsequent myotomy in a selected inner circular muscle was performed starting $2 \mathrm{~cm}$ caudally from the mid-esophageal incision up to $1-2 \mathrm{~cm}$ beyond the LES. The length of myotomy was $7-10 \mathrm{~cm}$. All procedures were 


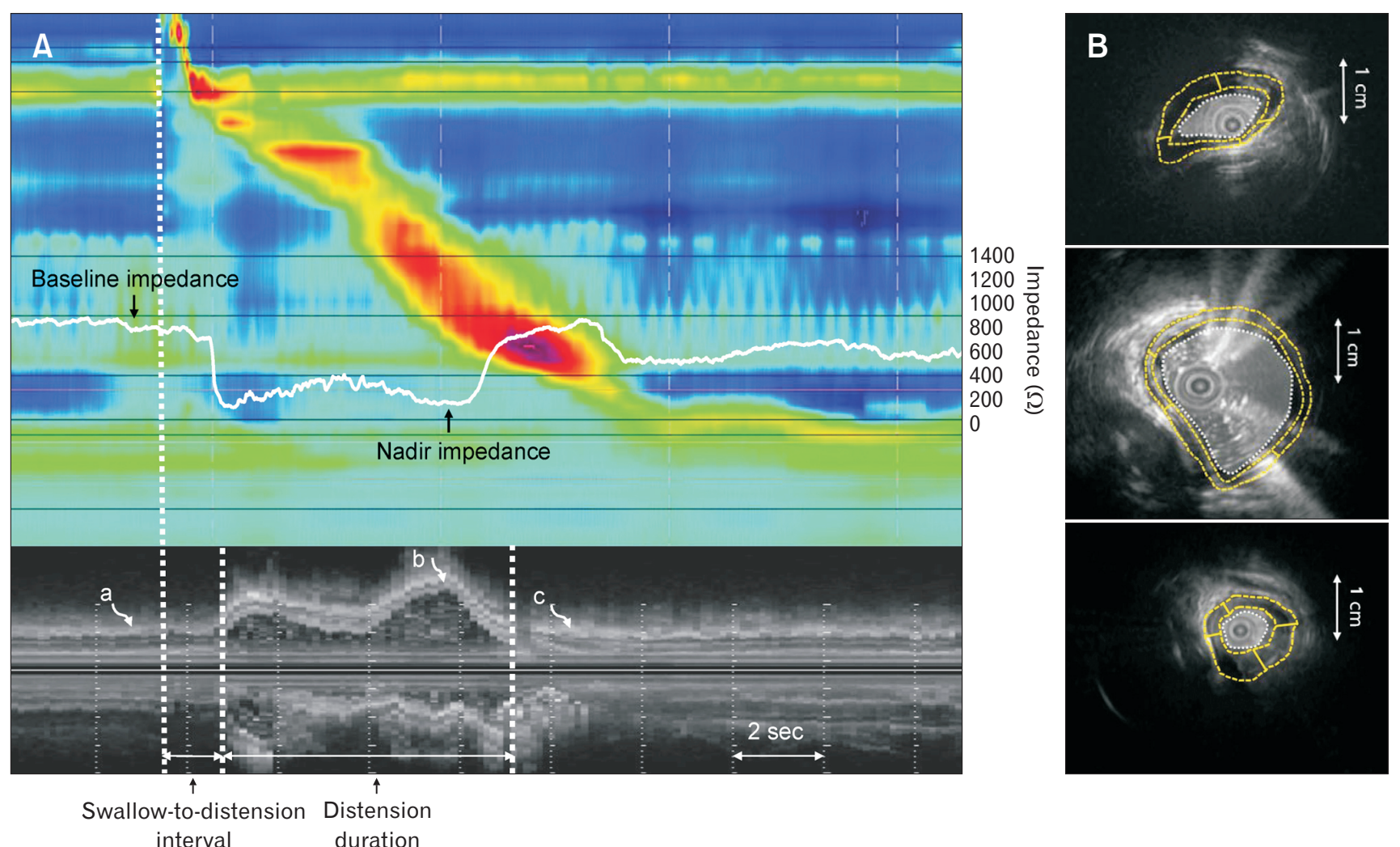

Figure 1. Intraluminal ultrasound (US) and impedance parameters in a healthy subject. (A) The impedance value $5 \mathrm{~cm}$ proximal to the lower esophageal sphincter and M-mode US images temporally correlated to obtain baseline and nadir impedance values. The swallow-to-distension interval and distension duration were calculated using M-mode images. The B-mode images for analyzing US parameters were selected using Mmode images (curved arrows a, b, and c). (B) The baseline/contraction/distension muscle thickness, muscle cross-sectional area, and lumen crosssectional area were analyzed using the corresponding B-mode US images.

performed using a hook knife (Olympus) and the spray coagulation mode at $60 \mathrm{~W}$ on effect 2 (ERBE, Tübingen, Germany).

Pneumatic dilation was performed under fluoroscopic guidance with the use of a 20-30-mm Rigiflex dilater (Boston Scientific, Boston, MA, USA). The dilater was inflated with a maximum of 15 psi for 60 seconds and repeated 2 times.

All examinations and procedures were performed by a single experienced investigator (J.H.K.).

\section{Statistical Methods}

Data are reported as the mean ( \pm standard deviation) or median (interquartile range 25-75) when appropriate. The baseline characteristics and US images and luminal impedance parameters of the healthy controls and patients were compared by chi-square test, independent $t$ test and one-way ANOVA. A paired $t$ test was employed to compare US images and luminal impedance parameters (MT, MCSA, LCSA, contractility/distensibility index, swallow-to- distension interval, distension duration, and baseline/nadir impedance value) before and after POEM or PD. A multivariate analysis was conducted with a binary logistic regression test. A $P$-value $<$ 0.05 was considered statistically significant.

\section{Ethical Approval}

This study was approved by the Institutional Review Board of Inje University Busan Paik Hospital (Approval No. 19-0106), which confirmed that the study was performed in accordance with the ethical guidelines of the Helsinki Declaration.

All authors had access to the study data and reviewed and approved the final manuscript. 


\section{Results}

\section{Baseline Characteristics: Peroral Endoscopic Myotomy Versus Pneumatic Dilatation Group}

Among 83 patients who were diagnosed with achalasia, 68 underwent POEM or PD as an initial therapeutic modality and had pre- and post-procedural intraluminal US performed simultaneously with HRIM. Among them, 3 patients who underwent PD were excluded because of treatment failure, 6 patients ( 2 underwent POEM and 4 underwent PD) were excluded because of loss to follow-up for clinical outcomes within a year, and 12 patients were excluded because the US images were too poor to analyze. Finally, 47 patients (30 and 17 patients in the POEM and PD groups, respectively, with a mean age of 50.11 [22-76] years and mean follow-up period of 22.34 [12-38] months), fulfilled the inclusion criteria. There was no significant difference between the POEM and $\mathrm{PD}$ groups in terms of sex, age, BMI, follow-up period, type of achalasia, Eckardt score, pre- and post-procedural 4s-IRP, or basal LES pressure. The frequency of PEP was also not different between the POEM and PD groups (40 in type II, 3 in type I vs 32 in type II, 3 in type I).

The symptom relapse rate during the follow-up period was higher in the PD group ( 2 and 4 cases in type II and III achalasia, respectively) than in the POEM group ( 1 case in type II and 1 case in type III achalasia, respectively) $(35.26 \%$ vs $6.67 \%, P=0.041)$ (Table 1).

The US images and impedance parameters of 40 swallow samples from healthy controls and 218 swallow samples from 47 achalasia patients ( 53 swallows from type I, 82 from type II, and 83 from type III) were analyzed. No significant differences were found among type I, II, and III achalasia patients in terms of age or follow-up duration. BMI was significantly higher in type III achalasia patients than in type I and II achalasia patients. Bolus transport was complete in $96.36 \%$ and sequential in $73.49 \%$ of analyzed swallows from type III achalasia patients (Table 2).

Post-procedural US images and the impedance parameters of 218 swallows were obtained from the 47 achalasia patients.

\section{Baseline High-resolution Impedance Manometry, Ultrasound Images, and Impedance Parameters According to Subtype of Achalasia}

The baseline, distension, and contraction MT values were largest in type III achalasia, followed by type II and type I achalasia $(P<0.001)$. Distension MT was smaller than baseline MT in healthy controls and type I and III achalasia patients $(P<0.001)$. In contrast, distension MT was larger than baseline MT in type II

Table 1. Clinical and Manometric Parameters of Patients Who Underwent Peroral Endoscopic Myotomy and Pneumatic Dilatation

\begin{tabular}{|c|c|c|c|}
\hline Parameters & $\operatorname{POEM}(\mathrm{n}=30)$ & $\operatorname{PD}(\mathrm{n}=17)$ & $P$-value \\
\hline Sex (male/female) & $15 / 15$ & $7 / 10$ & 0.564 \\
\hline Age (yr) & $51.03 \pm 15.13$ & $48.47 \pm 16.36$ & 0.591 \\
\hline $\operatorname{BMI}\left(\mathrm{kg} / \mathrm{m}^{2}\right)$ & $21.90 \pm 3.22$ & $22.05 \pm 4.08$ & 0.892 \\
\hline Follow-up period (mo) & $20.67 \pm 7.95$ & $25.29 \pm 8.96$ & 0.074 \\
\hline Type of achalasia & & & 0.948 \\
\hline Type I & $8(26.7)$ & $4(23.5)$ & \\
\hline Type II & $10(33.3)$ & $7(41.2)$ & \\
\hline Type III & $12(40.0)$ & $6(35.3)$ & \\
\hline \multicolumn{4}{|l|}{ Pre-procedural parameters } \\
\hline Eckardt score & $6.90 \pm 1.67$ & $6.82 \pm 0.81$ & 0.834 \\
\hline 4s-IRP (mmHg) & $33.68 \pm 13.35$ & $33.02 \pm 14.59$ & 0.875 \\
\hline Basal LES pressure (mmHg) & $45.85 \pm 11.46$ & $40.16 \pm 12.8$ & 0.124 \\
\hline PEP (n/total swallows [\%]) & $43 / 133(32.3)$ & $35 / 85(41.18)$ & 0.365 \\
\hline \multicolumn{4}{|l|}{ Post-procedural parameters } \\
\hline 4s-IRP (mmHg) & $10.53 \pm 3.84$ & $12.71 \pm 4.27$ & 0.079 \\
\hline Basal LES pressure (mmHg) & $11.98 \pm 4.19$ & $12.58 \pm 4.83$ & 0.652 \\
\hline $\operatorname{PEP}(\mathrm{n} /$ total swallows $[\%])$ & $3 / 133(2.26)$ & $6 / 85(7.06)$ & 0.098 \\
\hline Symptom relapse & $2(6.67)$ & $6(35.26)$ & 0.041 \\
\hline
\end{tabular}

POEM, peroral endoscopic myotomy; PD, pneumatic dilatation; BMI, body mass index; 4s-IRP, 4-second integrated relaxation pressure; LES, lower esophageal sphincter; PEP, panesophageal pressurization.

Data are expressed as $\mathrm{n} / \mathrm{n}$, mean $\pm \mathrm{SD}$, or $\mathrm{n}(\%)$. 
Table 2. Baseline Clinical, Manometric, and Intraluminal Ultrasound Parameters of Healthy Controls and Achalasia Patients

\begin{tabular}{|c|c|c|c|c|c|}
\hline Parameters & Healthy control $(\mathrm{n}=8)$ & Type I $(\mathrm{n}=12)$ & Type II $(\mathrm{n}=17)$ & Type III ( $\mathrm{n}=18)$ & $P$-value \\
\hline Sex (male/female) & $3 / 5$ & $6 / 6$ & $8 / 9$ & $8 / 10$ & 0.889 \\
\hline Age (yr) & $45.13 \pm 10.56$ & $46.17 \pm 17.92$ & $47.12 \pm 15.39$ & $55.56 \pm 12.83$ & 0.195 \\
\hline $\operatorname{BMI}\left(\mathrm{kg} / \mathrm{m}^{2}\right)$ & $23.66 \pm 1.88$ & $20.12 \pm 1.82$ & $20.56 \pm 2.02$ & $24.50 \pm 4.04$ & $<0.001$ \\
\hline Eckardt score & & $6.83 \pm 1.19$ & $7.00 \pm 1.54$ & $6.78 \pm 1.48$ & 0.896 \\
\hline Followup period (month) & & $24.50 \pm 8.99$ & $19.59 \pm 7.66$ & $23.50 \pm 8.79$ & 0.242 \\
\hline $\operatorname{IRP}(\mathrm{mmHg})$ & & $32.15 \pm 13.97$ & $30.28 \pm 13.05$ & $37.30 \pm 13.82$ & 0.299 \\
\hline Basal LES pressure (mmHg) & & $39.27 \pm 11.10$ & $38.84 \pm 10.16$ & $51.49 \pm 10.97$ & 0.002 \\
\hline Complete bolus transport ( $\mathrm{n} /$ total sw & s $[\%])$ & $0 / 53(0)$ & $0 / 82(0)$ & $80 / 83(96.39)$ & $<0.001$ \\
\hline Sequential bolus transport (n/total sv & vs $[\%])$ & $0 / 53(0)$ & $0 / 82(0)$ & $61 / 83(73.49)$ & $<0.001$ \\
\hline \multicolumn{6}{|l|}{ Muscle thickness (mm) } \\
\hline Baseline & $1.75 \pm 0.22$ & $1.49 \pm 0.33$ & $2.08 \pm 0.31$ & $4.62 \pm 1.21$ & $<0.001$ \\
\hline Distension & $1.45 \pm 0.11$ & $1.37 \pm 0.21$ & $2.42 \pm 0.53$ & $4.08 \pm 1.35$ & $<0.001$ \\
\hline Contraction & $2.38 \pm 0.41$ & $1.51 \pm 0.32$ & $2.77 \pm 0.63$ & $4.77 \pm 1.20$ & $<0.001$ \\
\hline \multicolumn{6}{|l|}{ Muscle CSA $\left(\mathrm{mm}^{2}\right)$} \\
\hline Baseline & $124.37 \pm 14.51$ & $113.84 \pm 4.00$ & $230.95 \pm 54.99$ & $249.28 \pm 115.48$ & $<0.001$ \\
\hline Distension & $153.07 \pm 10.10$ & $139.63 \pm 10.92$ & $342.87 \pm 70.73$ & $369.19 \pm 184.53$ & $<0.001$ \\
\hline Contraction & $85.52 \pm 8.04$ & $118.18 \pm 17.81$ & $226.66 \pm 79.96$ & $282.41 \pm 109.63$ & $<0.001$ \\
\hline \multicolumn{6}{|l|}{ Lumen CSA $\left(\mathrm{mm}^{2}\right)$} \\
\hline Baseline & $75.93 \pm 7.30$ & $625.86 \pm 145.97$ & $597.31 \pm 129.40$ & $59.93 \pm 1.99$ & $<0.001$ \\
\hline Distension & $321.00 \pm 47.97$ & $700.37 \pm 106.27$ & $683.90 \pm 109.51$ & $147.38 \pm 6.12$ & $<0.001$ \\
\hline Contraction & $48.89 \pm 5.77$ & $620.03 \pm 77.91$ & $428.26 \pm 95.52$ & $51.05 \pm 5.51$ & $<0.001$ \\
\hline Contractility index & $0.84 \pm 0.32$ & $0.091 \pm 0.063$ & $0.43 \pm 0.10$ & $0.65 \pm 0.33$ & $<0.001$ \\
\hline Distensibility index & $4.24 \pm 0.62$ & $1.14 \pm 0.11$ & $1.16 \pm 0.15$ & $2.17 \pm 0.68$ & $<0.001$ \\
\hline Baseline impedance $(\Omega)$ & $921.75 \pm 118.82$ & $168.68 \pm 33.14$ & $211.07 \pm 88.10$ & $1747.19 \pm 461.58$ & $<0.001$ \\
\hline Nadir impedance $(\Omega)$ & $180.50 \pm 47.18$ & & & $573.78 \pm 196.84$ & $<0.001$ \\
\hline Swallow-to-distension interval (sec) & $1.39 \pm 0.14$ & & & $2.25 \pm 0.35$ & $<0.001$ \\
\hline Distension duration (sec) & $6.24 \pm 0.53$ & & & $3.25 \pm 0.67$ & $<0.001$ \\
\hline
\end{tabular}

BMI, body mass index; IRP, integrated relaxation pressure; LES, lower esophageal sphincter; CSA, cross-sectional area.

Data are expressed as $n / n$ or mean $\pm S D$.

achalasia $(P<0.001)$.

In regard to the MCSA, the baseline, distension, and contraction MCSA values were significantly increased in type II and type III achalasia compared to the healthy controls and type I achalasia, but no significant difference was found between type II and type III achalasia $(P<0.001)$.

The baseline, distension, and contraction LCSA values significantly increased in type I and type II achalasia compared to the normal control. In type III achalasia, the baseline and contraction LCSA values were not significantly different from those in the healthy controls, but the distension LCSA was significantly decreased compared to the healthy controls $(P<0.001)$.

The contractility index was largest in healthy controls, followed by type III, type II, and type I achalasia. The distensibility index in type I, II and III achalasia was significantly smaller than that in the healthy controls. Both indices were significantly larger in type III achalasia than in type I and II achalasia, and no significant difference was found between type I and II achalasia.

The baseline luminal impedance level significantly decreased in type I and type II achalasia compared to the healthy controls, which is concordant with the baseline LCSA results. In contrast, the baseline luminal impedance was significantly greater in type III achalasia than in type I and II achalasia and in the healthy controls $(P<$ 0.001). The nadir luminal impedance level in type III achalasia also increased compared with that in the healthy controls $(P<0.001)$.

The swallow-to-distension interval was significantly prolonged and the distension duration was significantly decreased in type III achalasia patients compared to healthy controls (Table 2).

\section{Type I Achalasia: Post-peroral Endoscopic Myotomy and Pneumatic Dilatation}

The nadir impedance value was not identified, and bolus clear- 
MT (mm)

A

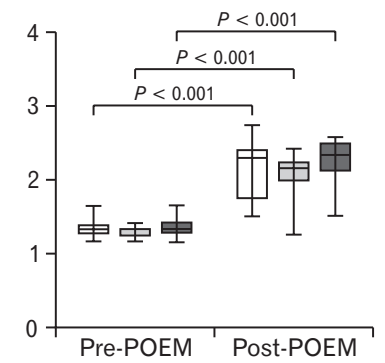

B
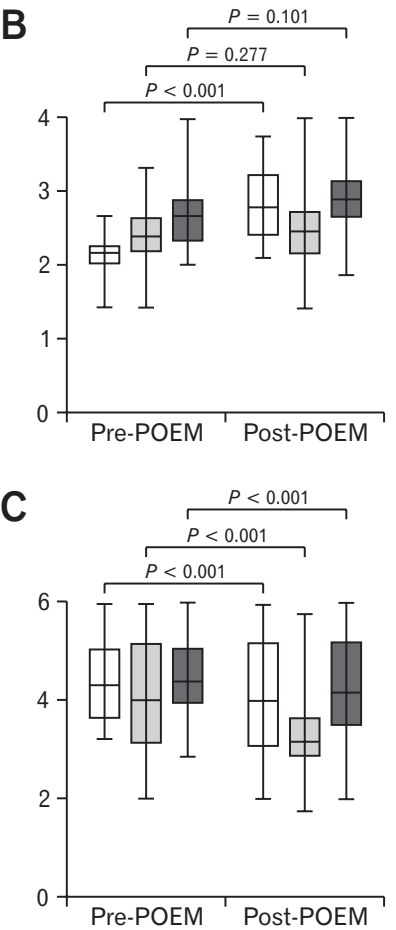

$\operatorname{MCSA}\left(\mathrm{mm}^{2}\right)$
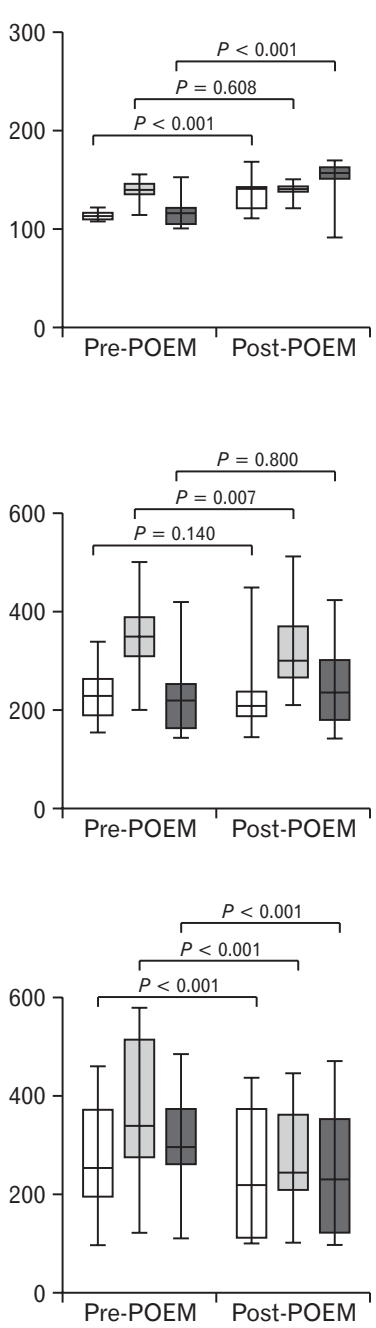

$\operatorname{LCSA}\left(\mathrm{mm}^{2}\right)$
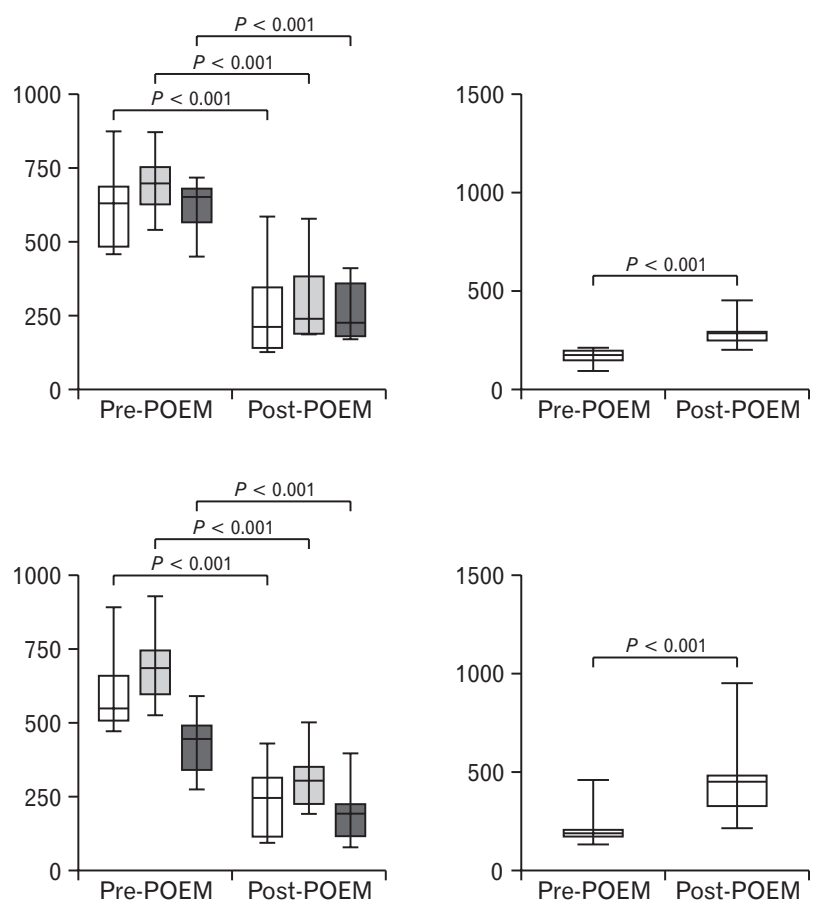

Impedance value $(\Omega)$

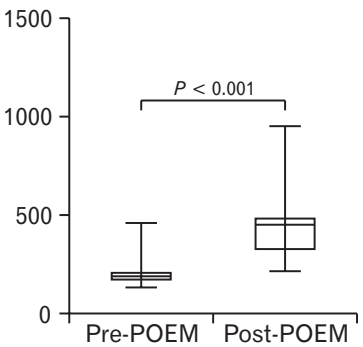

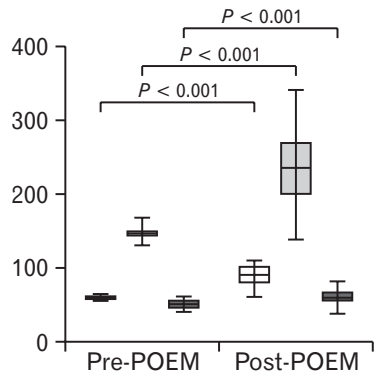

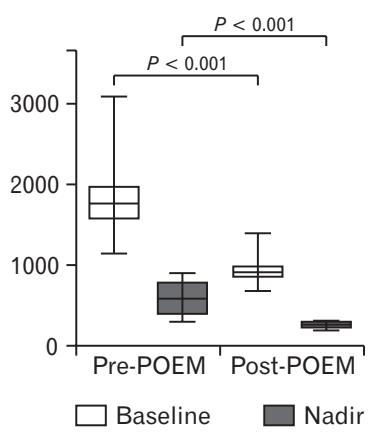

Figure 2. Changes in the muscle thickness (MT), muscle cross-sectional area (MCSA), lumen cross-sectional area (LCSA), and impedance values after peroral endoscopic myotomy (POEM) in type I (A), type II (B), and type III achalasia (C). The MT, baseline/contraction MCSA, and baseline impedance significantly increased in type I achalasia. Baseline MT and baseline impedance significantly increased in type II achalasia. LCSA significantly decreased in type I and II achalasia. In contrast, the MT, MCSA, and baseline/nadir impedance significantly decreased, and the LCSA significantly increased in type III achalasia.

ance was incomplete in $100 \%$ of swallows after POEM and PD.

The baseline, distension, and contraction MT values significantly increased after POEM and PD $(P<0.001)$. The baseline and contraction MCSA values also significantly increased $(P<$ 0.001), but the distension MCSA did not significantly changed after POEM and PD compared with pre-POEM and PD $(P=$ 0.608 and $P=0.347$, respectively).

In regard to the LCSA, the baseline, distension, and contrac- tion LCSA values significantly decreased in both the POEM and PD groups $(P<0.001)$. The baseline impedance level also significantly increased after POEM and PD $(P<0.001)$ (Fig. 2A and $3 A)$.

The distensibility index significantly increased after POEM $(1.14 \pm 0.10$ vs $1.20 \pm 0.15, P<0.001)$ and $\mathrm{PD}(1.15 \pm 0.13$ vs $1.23 \pm 0.19, P<0.001)$. However, the contractility index was not significantly changed in either the POEM or PD group $(P=0.880$ 
MT (mm)

A

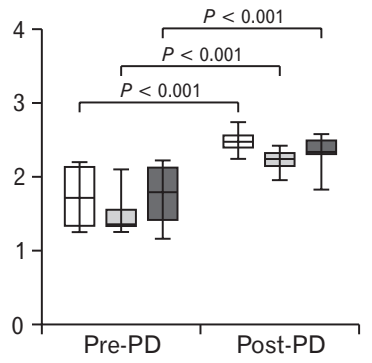

B

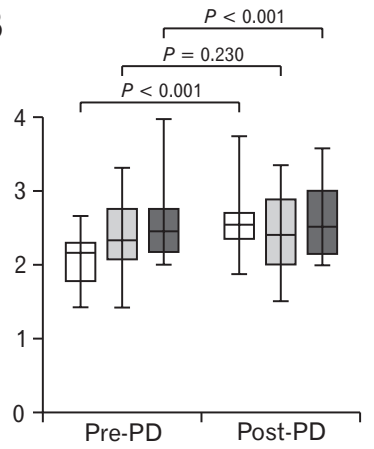

C

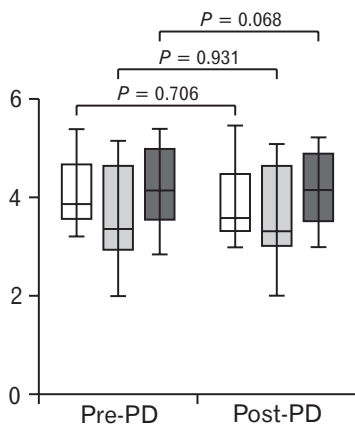

$\operatorname{MCSA}\left(\mathrm{mm}^{2}\right)$
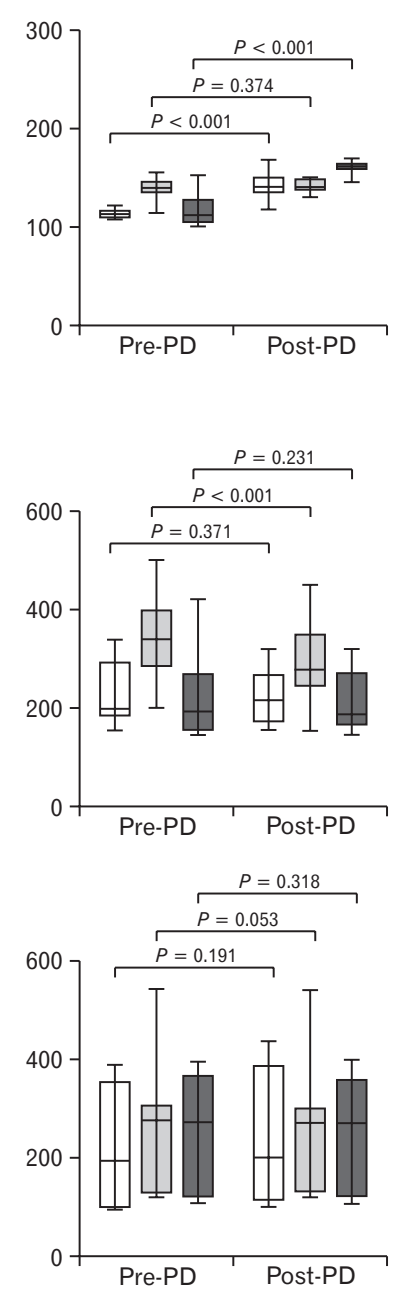

$\square$ Baseline
$\operatorname{LCSA}\left(\mathrm{mm}^{2}\right)$

Impedance value $(\Omega)$
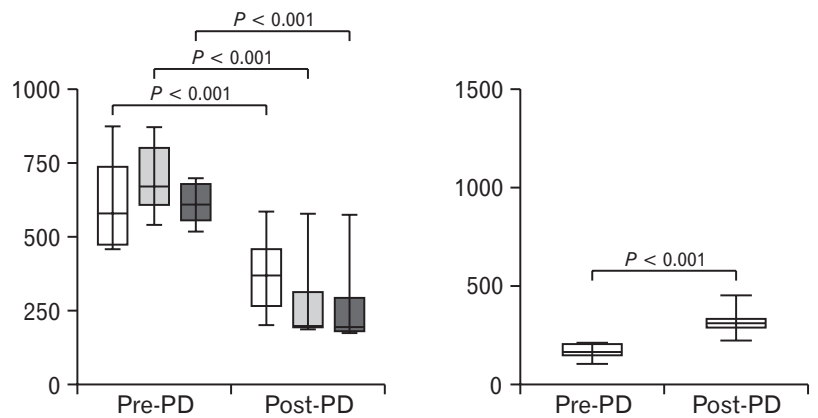
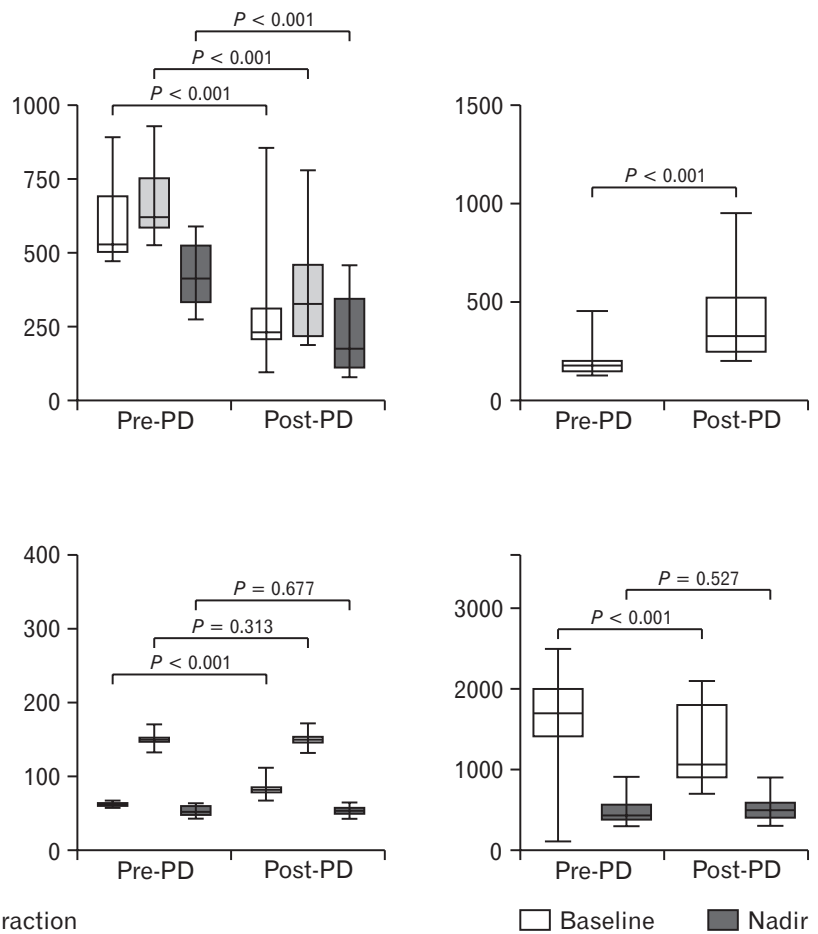

Figure 3. Changes in the muscle thickness (MT), muscle cross-sectional area (MCSA), lumen cross-sectional area (LCSA), and impedance values after pneumatic dilatation (PD) in type I (A), type II (B), and type III achalasia (C). As with the peroral endoscopic myotomy (POEM) group, the MT, baseline/contraction MCSA, and baseline impedance significantly increased in type I achalasia, and the LCSA significantly decreased in type I and II achalasia. The baseline LCSA and impedance significantly increased and decreased, respectively, in type III achalasia. However, the MT, MCSA, distension/contraction LCSA, and nadir impedance did not change significantly in type III achalasia.

and $P=0.141$, respectively) (Fig. $4 \mathrm{~A}$ and $4 \mathrm{~B}$ ).

\section{Type II Achalasia: Post-peroral Endoscopic Myotomy and Pneumatic Dilatation}

Simultaneous bolus transport with complete bolus clearance was observed in 7 swallows of 47 analyzed swallows (14.9\%) in the POEM group. Complete bolus clearance was found in 6 swallows of 35 analyzed swallows (17.1\%) (4 simultaneous and 2 sequential) in the PD group. PEP was observed in 3 and 6 swallows in the
POEM and PD groups, respectively.

The baseline MT values significantly increased after POEM and $\mathrm{PD}(P<0.001)$. However, there was no significant change in the distension MT in either the POEM or PD group $(P=0.277$ and $P=0.230$, respectively). The contraction MT values significantly increased in the PD group $(P<0.001)$ but unchanged in the POEM group $(P=0.101)$. Distension MT decreased than the baseline MT post-procedure in both the POEM and PD groups ( $P<0.001$ for each). 


\section{Contractility index Distensibility index}

A

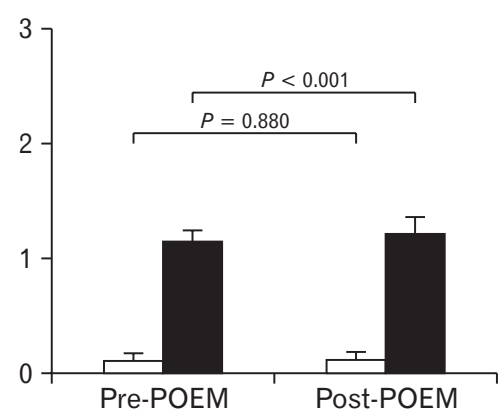

C

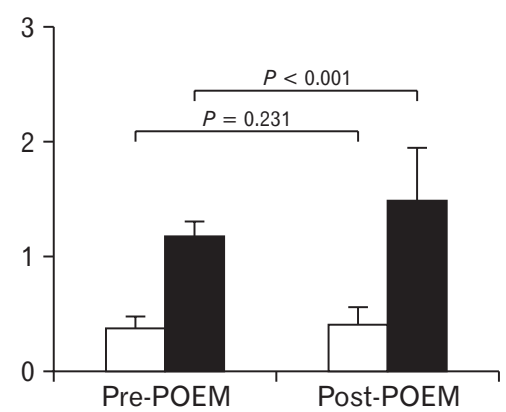

E

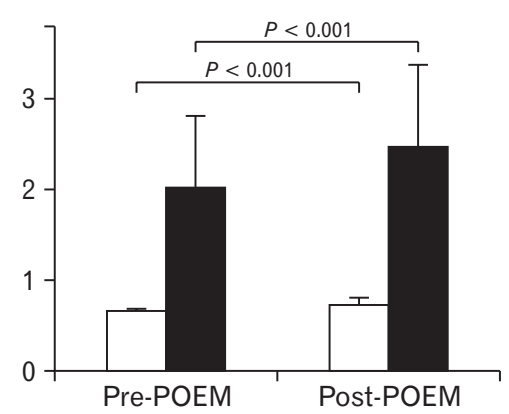

B 3

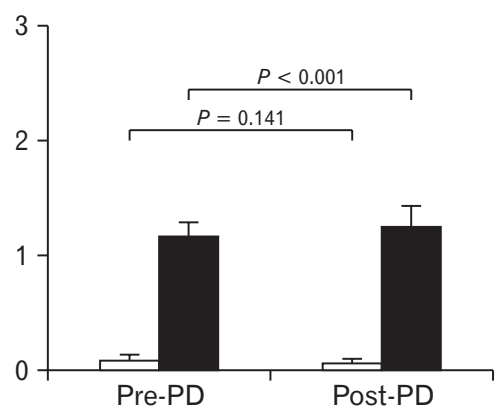

D

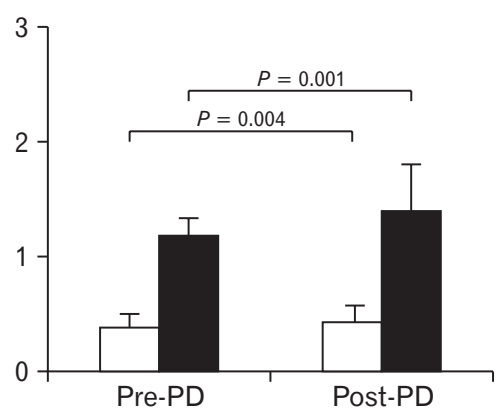

$\mathbf{F}$

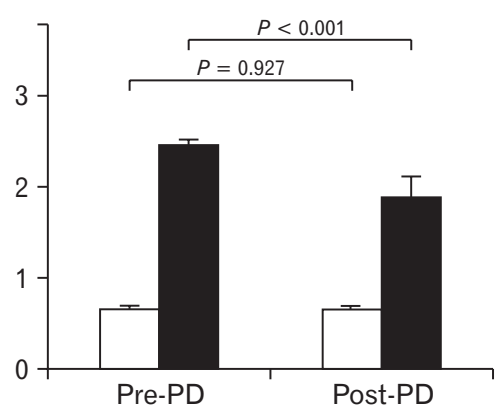

Figure 4. Changes in the contractility index and the distensibility index after peroral endoscopic myotomy (POEM) and pneumatic dilatation $(\mathrm{PD})$ in type I (A, B), type II (C, D), and type III achalasia $(\mathrm{E}, \mathrm{F})$. The contractility index increased significantly after PD in type II achalasia and after POEM in type III achalasia. The distensibility index significantly increased after POEM in type I, II, and III achalasia and after PD in type I and II achalasia.
There was no significant difference in the baseline or contraction MCSA in either the POEM or PD group. However, the distension MCSA significantly decreased in both the POEM and PD groups ( $P=0.007$ and $P<0.001$, respectively).

The baseline, distension, and contraction LCSA values significantly decreased after POEM and PD $(P<0.001)$. The baseline impedance level significantly increased in both groups $(P<0.001)$ (Fig. 2B and 3B).

The contraction index was not changed significantly in the POEM group $(P=0.231)$, but it significantly increased in the $\mathrm{PD}$ group $(0.37 \pm 0.12$ vs $0.42 \pm 0.15, P=0.004)$. The distensibility index significantly increased in both the POEM $(1.16 \pm 0.15$ vs $1.48 \pm 0.47, P<0.001)$ and $\mathrm{PD}$ groups $(1.17 \pm 0.17$ vs 1.39 $\pm 0.41, P=0.001)$ (Fig. $4 \mathrm{C}$ and $4 \mathrm{D}$ ).

\section{Type III Achalasia: Post-peroral Endoscopic Myotomy and Pneumatic Dilatation}

Bolus clearance was complete in 47 of 53 analyzed swallows (88.7\%), and bolus transport was sequential in 44 swallows (83.0\%) in the POEM group and complete in 28 of 30 analyzed swallows (93.3\%) and sequential in 23 swallows $(76.7 \%)$ in the PD group.

The baseline, distension, and contraction MT/MCSA values significantly decreased after POEM $(P<0.001)$ but did not change significantly in the PD group.

The baseline, distension, and contraction LCSA values significantly increased, and the baseline/nadir impedance values significantly decreased after POEM $(P<0.001)$ (Fig. 2C). The baseline LCSA and impedance values significantly increased and decreased, respectively, in the PD group $(P<0.001)$, but the distension/con- 

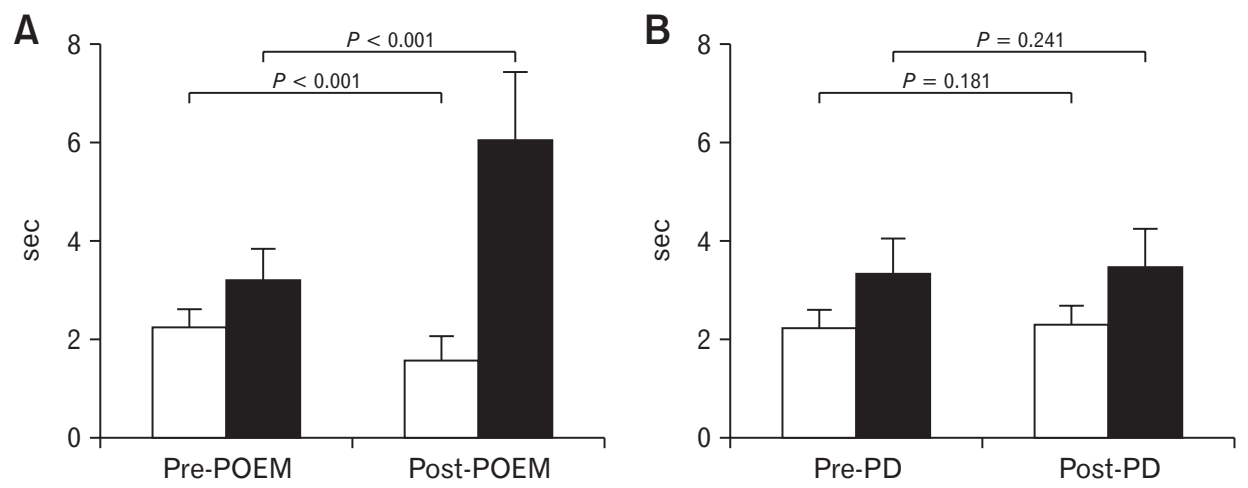

Figure 5. Changes in swallow-to-distension interval and distension duration after peroral endoscopic myotomy (POEM) and pneumatic dilatation (PD) in type III achalasia. The swallow-to-distension interval decreased, and the distension duration increased significantly after POEM. However, there was no significant change in either parameter after PD.

traction LCSA and nadir impedance values did not change significantly (Fig. 2C and 3C).

The contraction index significantly increased $(0.65 \pm 0.30 \mathrm{vs}$ $0.72 \pm 0.79, P<0.001)$, and the distensibility index also significantly increased $(2.09 \pm 0.80$ vs $2.47 \pm 0.89, P<0.001)$ in the POEM group. However, the distensibility index decreased significantly $(2.46 \pm 0.66$ vs $1.89 \pm 0.23, P<0.001)$, and the contractility index did not change significantly $(P=0.927)$ in the $\mathrm{PD}$ group (Fig. 4E and 4F).

The swallow-to-distension interval significantly decreased $(2.25 \pm 0.36$ vs $1.58 \pm 0.47, P<0.001)$, and the distension duration significantly increased $(3.20 \pm 0.47$ vs $6.04 \pm 1.39, P<0.001)$ after POEM. In contrast, there was no significant difference in either parameter in the PD group $(P=0.181$ and 0.241 , respectively) (Fig. 5A, 5B, and 6).

\section{Symptom Relapse After the Procedure in Type III Achalasia}

Four patients who underwent PD (66.7\%) and 1 patient who underwent POEM (8.3\%) experienced a relapse in symptoms during the follow-up period. Univariate analysis revealed that the post-procedural 4s-IRP, distension MT, distension MCSA, contraction MCSA, baseline/nadir impedance values, and swallow-todistension interval were significantly larger in relapsed patients than in non-relapsed patients. In contrast, the distension LCSA, distension duration, and contractility/distensibility index were significantly smaller in relapsed patients than in non-relapsed patients. Among those parameters, post-procedural distension LCSA $(P=0.002$; OR, $0.84 ; 95 \% \mathrm{CI}, 0.67-0.94)$ and distension duration $(P=0.041$; OR, 0.93 ; $95 \%$ CI, 0.54-0.98) remained significant factors for pre- dicting relapse in the multivariate analysis.

\section{Discussion}

In our study, type III achalasia was characterized by intact completeness and sequentiality in bolus transport, a markedly increased baseline and distension MT, and an insufficient distension LCSA, which are totally different characteristics from those in type I and II achalasia. Additionally, muscle thinning during the distension period of bolus transport, which is an important feature of the normal inhibitory process of primary peristalsis, ${ }^{15,19}$ was found to be insufficient in type III achalasia (Fig. 1 and 6). These findings are concordant with those of previous studies reporting inhibitory function abnormalities in esophageal body motility in type III achalasia. ${ }^{18,20}$

The morphological changes after POEM in type III achalasia can be summarized as decreases in the MT and MCSA and increases in the baseline and distension LCSA, which are distinctive changes from those in type I and II achalasia. POEM also improved the distensibility index despite the baseline LCSA increase. In other words, POEM physically improved esophageal body distensibility during bolus transit in type III achalasia. Additionally, we revealed that PD could not improve the intraluminal US parameters (except baseline LCSA) or the contractility/distensibility index in type III achalasia. These results may account for the superior effect of POEM compared with PD in type III achalasia. ${ }^{7,8}$

One of the unique findings of this study was that the prolonged swallow-to-distension interval and shortened distension duration, which are indicators of the inhibitory dysfunction of esophageal body peristalsis and assumed to be a cause of premature contraction, ${ }^{20}$ also improved after POEM but not after PD in type III 

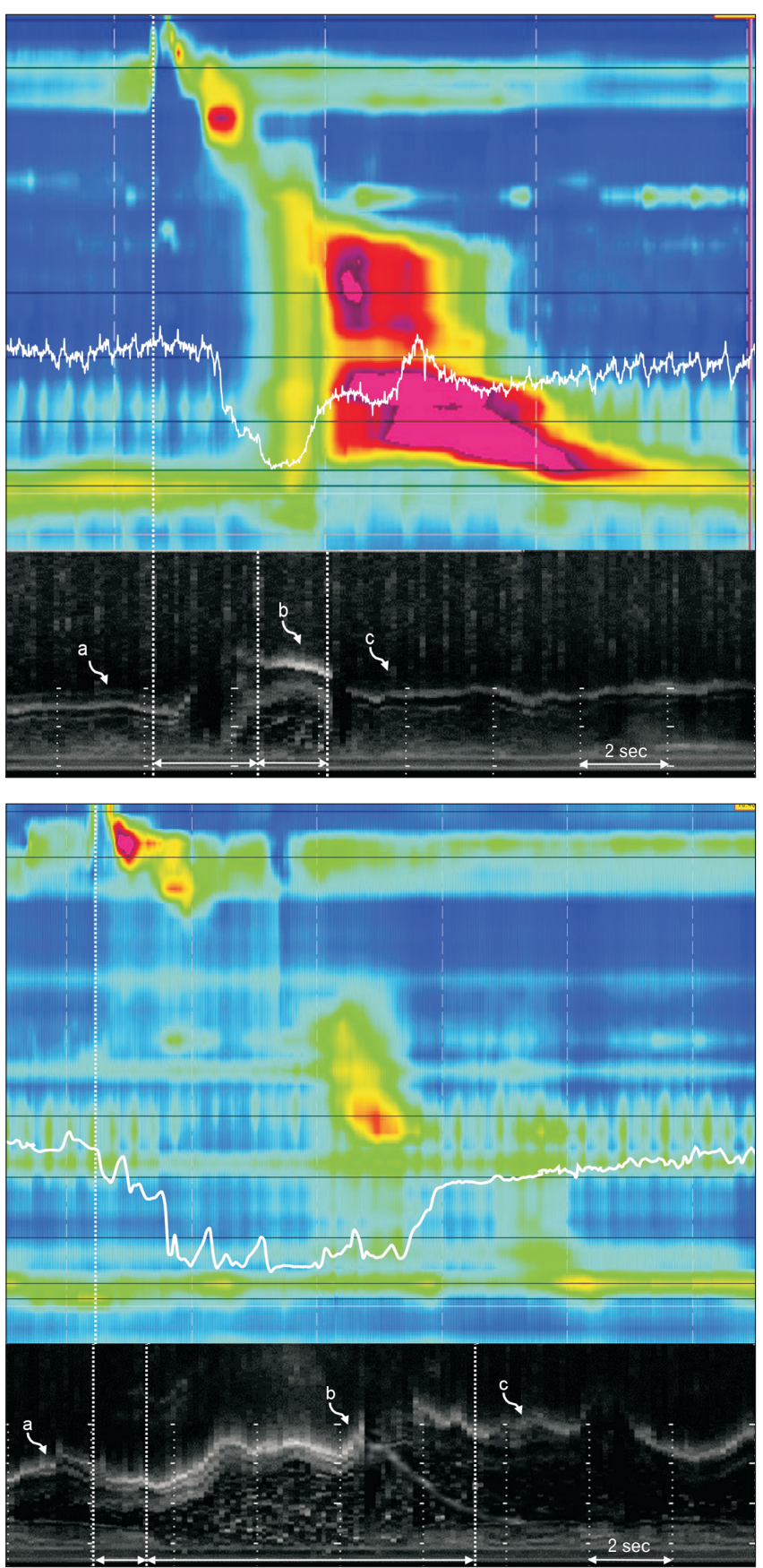

achalasia. Additionally, along the distension LCSA, post-procedural distension duration was revealed as a significant predicting factor for symptom relapse in type III achalasia. This also suggests that improving esophageal body compliance by long myotomy can improve the deglutitive inhibitory process during primary peristalsis and provides important clues regarding the mechanisms of symptom improvement after POEM in type III achalasia. As a result, our findings suggest that long myotomy is an essential component
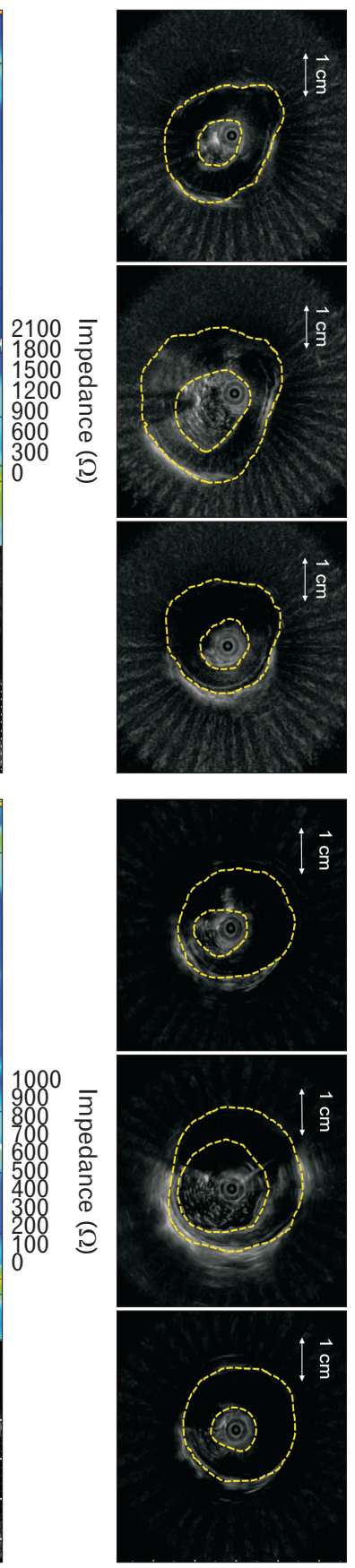

Figure 6. Changes in cross-sectional movement in the distal esophageal body after peroral endoscopic myotomy in type III achalasia at baseline, peak distension, and contraction points (curved arrows $a, b$, and c). The muscle thickness decreased, the lumen cross-sectional area, distension duration increased, and the swallow-to-distension interval decreased.

of POEM for type III achalasia management.

Our results also provide evidence supporting the application of POEM in other spastic esophageal motility disorders, such as jackhammer esophagus and distal esophageal spasm, in which inhibitory abnormalities in body peristalsis are prominent and LES relaxation is intact. ${ }^{11,21-24}$ Although additional controlled studies will be needed for verification, POEM may be a rational treatment strategy for these disorders. 
Along with esophageal body aperistalsis, increased muscle layer thickness is a well-known phenomenon in achalasia patients, ${ }^{25,26}$ and this is supposedly due to the sustained increase in intrabolus pressure with underlying esophageal myopathy. ${ }^{27,28}$ In addition, paradoxical muscle thickening during distension, which may be the cause of PEP, was found in most cases of type II achalasia (Supplementary Fig. 1).

Studies reporting changes in the muscle layer after normalizing the LES pressure are scarce. Sinn et $\mathrm{al}^{29}$ performed research to evaluate changes in the esophageal MCSA after PD and showed inconsistent results and no relationship with symptom recurrence. However, 2 points should be considered when interpreting the results: because the diagnosis of achalasia was made based on conventional manometry in that study, achalasia subtyping was not available, and data other than those for MCSA were not analyzed. Considering that the MCSA in achalasia is affected by the lumen $\mathrm{CSA}$ as well as the MT, ${ }^{25,30}$ the study lacks evidence estimating morphological changes in the esophageal body after LES decompression.

Our results showed that the baseline esophageal body MT increased in both type I and type II achalasia after POEM or PD. Especially in type I achalasia, the body MT became thicker than that of the normal controls, though it was thinner than that of the normal control before POEM or PD (Supplementary Fig. 2). These results may be explained by the pathologically thickened body muscle layer becoming prominent after POEM and PD, which effectively decreased the intrabolus pressure.

Our study shows that the distended LCSA dramatically decreased after POEM in type I and II achalasia due to an effective decrease in LES pressure, and these results were also observed in the PD group.

However, our results showed that POEM could not effectively improve lumen-reducing esophageal contractility in type I and II achalasia, and the contractility index was increased after PD in type II achalasia. Long myotomy resulting in a decrease in esophageal body contractility may be an explanation for these results. ${ }^{31,32}$ These findings also suggest that esophageal contractility abnormalities in type I achalasia may be irreversible even after effective LES decompression. ${ }^{33}$

Interestingly, our study shows that PEP, which was a characteristic finding of type II achalasia and accompanied by paradoxical muscle thickening during distension, disappeared in most cases of type II achalasia not only in the POEM group (Supplementary Fig. 1), but also in the PD group. Although it is still debated whether the unique esophageal body motility pattern is caused by primary myopathy of the esophageal body or is secondary to increased intrabolus pressure, our results suggest that effective LES decompression alone can normalize the PEP in type II achalasia. ${ }^{12}$

Based on our results in type I and II achalasia, we cautiously propose that long myotomy is not necessarily essential in type I and II achalasia and that POEM with short myotomy, which ensures complete LES myotomy, can be as effective as POEM with long myotomy. $^{34}$

Our study has several inevitable methodologic limitations.

Certain patients with type I and II achalasia (8 with type I achalasia and 4 with type II achalasia) who fulfilled the inclusion criteria were excluded because of unsuitable intraluminal US images (eg, the baseline and distension LCSAs were too large [more than 1000 $\left.\mathrm{mm}^{2}\right]$ to obtain whole images for analyzing the LCSA, or a large amount of remnant food was present in the esophageal lumen during the examination), which may have caused selection bias.

Principally, an intravascular US catheter and HRIM catheter should be assembled by taping the US transducer to the HRIM catheter before insertion into the patient to maintain the US transducer $5 \mathrm{~cm}$ proximal to the LES and the exact same level as the pressure/impedance senor of the HRIM catheter during the examination. ${ }^{35}$ However, we used an EUS catheter because (1) the intravascular US system was not available in our institute and (2) the US image defect induced by interference of the adjacent HRIM catheter was minimized. Instead, we located the US transducer by fixing the US catheter to the HRIM catheter outside the body of the subject using the positioning function of the HRIM program.

Our results may not represent the long-term morphologic changes in esophageal body movement because follow-up intraluminal US examination was performed 3-6 months after the procedure.

In conclusion, this study demonstrates that POEM decreased the esophageal LCSA by LES and intrabolus pressure decompression in type I and II achalasia. In contrast, POEM improved esophageal body distension and inhibitory processes during primary peristalsis in type III achalasia.

\section{Supplementary Materials}

Note: To access the supplementary figures mentioned in this article, visit the online version of Journal of Neurogastroenterology and Motility at http://www.jnmjournal.org/, and at https://doi. org/10.5056/jnm21020.

\section{Acknowledgements: None.}




\section{Financial support: None.}

\section{Conflicts of interest: None.}

Author contributions: Ji Hyun Kim: proposal of study idea and development of study protocol, subjects enrollment, performing examination and procedure, data analysis, and making manuscript; Alex Ju Sung Kim: development of image-analyzing software, data acquisition, statistical analysis, and making manuscript; Sungmoon Ong: data acquisition, statistical analysis, and making manuscript; Hong Sub Lee and Jun Sik Yoon: patient selection and data analysis; and Dae Young Hur: data analysis, review the study protocol, and data acquisition.

\section{References}

1. Kahrilas PJ, Bredenoord AJ, Fox M, et al. The Chicago classification of esophageal motility disorders, v3.0. Neurogastroenterol Motil 2015;27:160-174.

2. Pandolfino JE, Gawron AJ. Achalasia: a systematic review. JAMA 2015;313:1841-1852.

3. Sodikoff JB, Lo AA, Shetuni BB, Kahrilas PJ, Yang GY, Pandolfino JE. Histopathologic patterns among achalasia subtypes. Neurogastroenterol Motil 2016;28:139-145.

4. Salvador R, Costantini M, Zaninotto G, et al. The preoperative manometric pattern predicts the outcome of surgical treatment for esophageal achalasia. J Gastrointest Surg 2010;14:1635-1645.

5. Pratap N, Kalapala R, Darisetty S, et al. Achalasia cardia subtyping by high-resolution manometry predicts the therapeutic outcome of pneumatic balloon dilatation. J Neurogastroenterol Motil 2011;17:48-53.

6. Rohof WO, Salvador R, Annese V, et al. Outcomes of treatment for achalasia depend on manometric subtype. Gastroenterology 2013;144:718725 .

7. Ponds FA, Fockens P, Lei A, et al. Effect of peroral endoscopic myotomy vs pneumatic dilation on symptom severity and treatment outcomes among treatment-naive patients with achalasia: a randomized clinical trial. JAMA 2019;322:134-144.

8. Kim GH, Jung KW, Jung HY, et al. Superior clinical outcomes of peroral endoscopic myotomy compared with balloon dilation in all achalasia subtypes. J Gastroenterol Hepatol 2019;34:659-665.

9. Ngamruengphong S, Inoue $\mathrm{H}$, Ujiki MB, et al. Efficacy and safety of peroral endoscopic myotomy for treatment of achalasia after failed Heller myotomy. Clin Gastroenterol Hepatol 2017;15:1531-1537, e3.

10. Bechara R, Woo M, Hookey L, et al. Peroral endoscopic myotomy (POEM) for complex achalasia and the POEM difficulty score. Dig Endosc 2019;31:148-155.

11. Filicori F, Dunst CM, Sharata A, et al. Long-term outcomes following POEM for non-achalasia motility disorders of the esophagus. Surg Endosc 2019;33:1632-1639.

12. Roman S, Kahrilas PJ, Mion F, et al. Partial recovery of peristalsis after myotomy for achalasia: more the rule than the exception. JAMA Surg 2013;148:157-164.

13. Sanagapalli S, Plumb A, Maynard J, Leong RW, Sweis R. The timed barium swallow and its relationship to symptoms in achalasia: analysis of surface area and emptying rate. Neurogastroenterol Motil 2020;32:e13928.

14. Yoo IK, Choi SA, Kim WH, Hong SP, Cakir OO, Cho JY. Assessment of clinical outcomes after peroral endoscopic myotomy via esophageal distensibility measurements with the endoluminal functional lumen imaging probe. Gut Liver 2019;13:32-39.

15. Mittal RK, Liu J, Puckett JL, et al. Sensory and motor function of the esophagus: lessons from ultrasound imaging. Gastroenterology 2005;128:487-497.

16. Krishnan K, Lin CY, Keswani R, Pandolfino JE, Kahrilas PJ, Komanduri $\mathrm{S}$. Endoscopic ultrasound as an adjunctive evaluation in patients with esophageal motor disorders subtyped by high-resolution manometry. Neurogastroenterol Motil 2014;26:1172-1178.

17. Kim JH, Mittal RK, Patel N, Ledgerwood M, Bhargava V. Esophageal distension during bolus transport: can it be detected by intraluminal impedance recordings? Neurogastroenterol Motil 2014;26:1122-1130.

18. Kim TH, Patel N, Ledgerwood-Lee M, Mittal RK. Esophageal contractions in type 3 achalasia esophagus: simultaneous or peristaltic? Am J Physiol Gastrointest Liver Physiol 2016;310:G689-G695.

19. Yamamoto Y, Liu J, Smith TK, Mittal RK. Distension-related responses in circular and longitudinal muscle of the human esophagus: an ultrasonographic study. Am J Physiol 1998;275:G805-G811.

20. Park S, Zifan A, Kumar D, Mittal RK. Genesis of esophageal pressurization and bolus flow patterns in patients qith achalasia esophagus. Gastroenterology 2018;155:327-336.

21. Quader F, Mauro A, Savarino E, et al. Jackhammer esophagus with and without esophagogastric junction outflow obstruction demonstrates altered neural control resembling type 3 achalasia. Neurogastroenterol Motil 2019;31:e13678.

22. Wahba G, Bouin M. Jackhammer esophagus: a meta-analysis of patient demographics, disease presentation, high-resolution manometry data, and treatment outcomes. Neurogastroenterol Motil 2020;32:e13870.

23. Philonenko S, Roman S, Zerbib F, et al. Jackhammer esophagus: clinical presentation, manometric diagnosis, and therapeutic results-results from a multicenter French cohort. Neurogastroenterol Motil 2020;32:e13918.

24. Hernandez PV, Valdovinos LR, Horsley-Silva JL, Valdovinos MA, Crowell MD, Vela MF. Response to multiple rapid swallows shows impaired inhibitory pathways in distal esophageal spasm patients with and without concomitant esophagogastric junction outflow obstruction. Dis Esophagus 2020;33:1-6.

25. Mittal RK, Kassab G, Puckett JL, Liu J. Hypertrophy of the muscularis propria of the lower esophageal sphincter and the body of the esophagus in patients with primary motility disorders of the esophagus. Am J Gastroenterol 2003;98:1705-1712.

26. Dogan I, Puckett JL, Padda BS, Mittal RK. Prevalence of increased esophageal muscle thickness in patients with esophageal symptoms. Am J Gastroenterol 2007;102:137-145.

27. Gockel I, Bohl JR, Doostkam S, Eckardt VF, Junginger T. Spectrum of 
histopathologic findings in patients with achalasia reflects different etiologies. J Gastroenterol Hepatol 2006;21:727-733.

28. Puckett JL, Bhalla V, Liu J, Kassab G, Mittal RK. Oesophageal wall stress and muscle hypertrophy in high amplitude oesophageal contractions. Neurogastroenterol Motil 2005;17:791-799.

29. Sinn DH, Choi YS, Kim JH, et al. Change in cross-sectional area of esophageal muscle does not correlate with the outcome of achalasia after pneumatic balloon dilatation. J Gastroenterol Hepatol 2010;25:539-543.

30. Takeda T, Kassab G, Liu J, Puckett JL, Mittal RR, Mittal RK. A novel ultrasound technique to study the biomechanics of the human esophagus in vivo. Am J Physiol Gastrointest Liver Physiol 2002;282:G785-G793.

31. Ren Y, Tang X, Chen F, et al. Myotomy of Distal esophagus influences proximal esophageal contraction and upper esophageal sphincter relaxation in patients with achalasia after peroral endoscopic myotomy. J Neu- rogastroenterol Motil 2016;22:78-85.

32. Hu Y, Li M, Lu B, Meng L, Fan Y, Bao H. Esophageal motility after peroral endoscopic myotomy for achalasia. J Gastroenterol 2016;51:458464.

33. Hong SJ, Bhargava V, Jiang Y, Denboer D, Mittal RK. A unique esophageal motor pattern that involves longitudinal muscles is responsible for emptying in achalasia esophagus. Gastroenterology 2010;139:102-111.

34. Nabi Z, Ramchandani M, Sayyed M, et al. Comparison of short versus long esophageal myotomy in cases with idiopathic achalasia: a randomized controlled trial. J Neurogastroenterol Motil 2021;27:63-70.

35. Leslie E, Bhargava V, Mittal RK. A novel pattern of longitudinal muscle contraction with subthreshold pharyngeal stimulus: a possible mechanism of lower esophageal sphincter relaxation. Am J Physiol Gastrointest Liver Physiol 2012;302:G542-G547. 